\title{
A Survey on Cooperative Communication in Wireless Networks
}

\author{
A. F. M. Shahen Shah \\ Institute of Information and Communication Technology, Bangladesh University of Engineering and Technology, \\ Dhaka, Bangladesh \\ Email: mit1002@iit.du.ac.bd, shahen.shah@hotmail.com
}

Md. Shariful Islam

Institute of Information Technology, University of Dhaka, Dhaka-1000, Bangladesh

Email: shariful@univdhaka.edu

\begin{abstract}
Cooperative communication in wireless networks has become more and more attractive recently since it could mitigate the particularly severe channel impairments arising from multipath propagation. Here the greater benefits gained by exploiting spatial diversity in the channel. In this paper, an overview on cooperative communication in wireless networks is presented. We inscribe the benefits of cooperative transmission than traditional non - cooperative communication. Practical issues and challenges in cooperative communication are identified. In particular, we present a study on the advantages, applications and different routing strategies for cooperative mesh networks, Ad hoc networks and wireless sensor networks.
\end{abstract}

Index Terms- MIMO, Cooperative Communication, Cooperative Mesh Network, Cooperative Ad Hoc Network, Cooperative Sensor Network, Relaying.

\section{INTRODUCTION}

Transmit diversity generally requires more than one antenna at the transmitter. However, many wireless devices are limited by size or hardware complexity to one antenna. Recently, a new class of methods called cooperative communication has been proposed that enables single antenna mobiles in a multi-user environment to share their antennas and generate a virtual multiple-antenna transmitter that allows them to achieve transmit diversity.

The advantages of multiple-input multiple-output (MIMO) systems have been widely acknowledged, to the extent that certain transmit diversity methods (i.e., Alamouti signaling) have been incorporated into wireless standards. Although transmit diversity is clearly advantageous on a cellular base station, it may not be practical for other scenarios. Specifically, due to size, cost, or hardware limitations, a wireless agent may not be able to support multiple transmit antennas. Examples include most handsets (size) or the nodes in a wireless sensor network (size, power).

Cooperative communication allows single-antenna mobiles to reap some of the benefits of MIMO systems. The basic idea is that single-antenna mobiles in a multiuser scenario can "share" their antennas in a manner that creates a virtual MIMO system. Several important milestones in this area have been achieved, leading to a flurry of further research activity. It is our hope that this article will serve to illuminate the subject for a wider audience, and thus accelerate the pace of developments in this exciting technology.

The mobile wireless channel suffers from fading [1], meaning that the signal attenuation can vary significantly over the course of a given transmission. Transmitting independent copies of the signal generates diversity and can effectively combat the deleterious effects of fading. In particular, spatial diversity is generated by transmitting signals from different locations, thus allowing independently faded versions of the signal at the receiver. Cooperative communication generates this diversity in a new and interesting way [2].

With the advantage of broadcast in wireless medium, cooperative communication is proposed recently [2], which allows several nodes cooperatively transmit signals to a destination together. Researches have shown that cooperative communication can offer significant performance enhancements in terms of increased capacity, improved transmission reliability, spatial diversity and diversity-multiplexing tradeoff [3]. In [48], the authors present an overview of cooperative communications. To the best of our knowledge, reference [4] is the first work discussed cooperative communication aware routing. In this work, the authors investigated the energy efficient routing which supports broadcast and cooperative communication. The recent works by Shoukang Zheng [5] which gives the performance study of cooperative routing metric for multi-hop wireless networks. Most recent works by A. F. M. Shahen Shah et al [52] present a routing metric for efficient cooperative communication. Stark C. Draper et al. [6] show a study on cooperative transmission for wireless networks using mutualinformation accumulation. In [49], the authors survey cooperative communication schemes and discuss their advantages in improving system capacity and diversity. Then they examine the applications of cooperative relaying schemes in LTE-advanced systems. Mohamed Elhawary et al. [7] propose an energy-efficient protocol for cooperative networks. In [8], authors consider the problem of sending information packets from a source 
node to a destination node using a network of cooperating wireless relays to minimize the total energy consumption. Cooperative routing provides efficiency in Ad hoc networks, wireless mesh networks and wireless sensor networks. Min Sheng et al. [9] give small world based cooperative routing protocol for large scale wireless Ad Hoc networks. Recently Zhiguo Ding et al. [10], CrossLayer Routing is studied Using Cooperative Transmission in Vehicular Ad-hoc Networks. Youngchul Sung et al. [11] provide cooperative routing for distributed detection in large sensor networks. A crosslayer cooperative method for IEEE 802.16 mesh networks is suggested by [12]. Christian Ibars et al. [13] increase the throughput [14] of wireless mesh networks with cooperative techniques.

However, we provide an overview on cooperative communication and comparison with traditional noncooperative communication. Advantages, applications, routing strategies for cooperative wireless mesh networks, Ad hoc networks and wireless sensor networks are also presented in this paper.

The rest of the paper is organized as follows. In Section II, an overview of Cooperative Communication is given. In Section III, Cooperative routing in Wireless Mesh Networks is described. The Cooperative routing strategies in ad hoc networks is carried out in Section IV. Cooperative Routing in Wireless Sensor Networks is presented in Section V. Finally, we conclude the paper in Section VI.

\section{COOPERATIVE COMMUNICATION: AN OVERVIEW}

\subsection{Cooperative Communication}

With the advantage of broadcast in wireless medium, cooperative communication is proposed recently [2], which allows several nodes cooperatively transmit signals to a destination together. Researches have shown that cooperative communication can offer significant performance enhancements in terms of increased capacity, improved transmission reliability, spatial diversity and diversity-multiplexing tradeoff [16-19].

Cooperative communication typically refers to a system where users share and coordinate their resources to enhance the information transmission quality. It is a generalization of the relay communication, in which multiple sources also serve as relays for each other [50]. For a preliminary explanation of the ideas behind cooperative communication, we refer the reader to Fig. 1. This figure shows two mobile agents communicating with the same destination. Each mobile has one antenna and cannot individually generate spatial diversity. However, it may be possible for one mobile to receive the other, in which case it can forward some version of "overheard" information along with its own data. Because the fading paths from two mobiles are statistically independent, this generates spatial diversity.

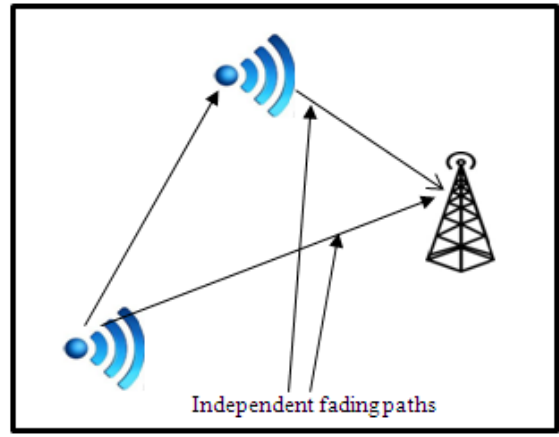

Fig. 1. Cooperative communication

In the figures we use icons resembling base stations or handsets, but this is only a convenient graphical representation. The idea of cooperation is general, and perhaps even more suitable to ad hoc wireless networks and wireless sensor networks than cellular networks.

In cooperative wireless communication, we are concerned with a wireless network, of the cellular or ad hoc variety, where the wireless agents, which we call users, may increase their effective quality of service (measured at the physical layer by bit error rates, block error rates, or outage probability) via cooperation.

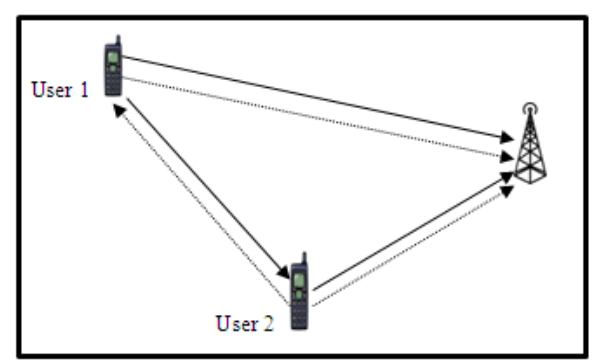

Fig. 2. In cooperative communication each mobile is both a user and a relay

In a cooperative communication system, each wireless user is assumed to transmit data as well as act as a cooperative agent for another user (Fig. 2). In a relay system, sources first transmit their data to the relay nodes (RNs). Each RN then processes and forwards its received data information to the destination nodes following some cooperation protocols. With the received signal from the RNs, the destinations decode the data from their corresponding sources [49].

Cooperation leads to interesting trade-offs in code rates and transmit power. In the case of power, one may argue on one hand that more power is needed because each user, when in cooperative mode, is transmitting for both users. On the other hand, the baseline transmits power for both users will be reduced because of diversity. In the face of this trade-off, one hopes for a net reduction of transmit power, given everything else being constant.

Similar questions arise for the rate of the system. In cooperative communication each user transmits both his/her own bits as well as some information for his/her partner; one might think this causes loss of rate in the system. However, the spectral efficiency of each user improves because; due to cooperation diversity the channel code rates can be increased. Again a tradeoff is 
observed. The key question, whether cooperation is worth the incurred cost, has been answered positively by several studies.

One may also describe cooperation as a zerosum game in terms of power and bandwidth of the mobiles in the network. The premise of cooperation is that certain (admittedly unconventional) allocation strategies for the power and bandwidth of mobiles lead to significant gains in system performance. In the cooperative allocation of resources, each mobile transmits for multiple mobiles [2].

\subsection{Historical Background}

The basic ideas behind cooperative communication can be traced back to the groundbreaking work of Cover and El Gamal on the information theoretic properties of the relay channel [16]. This work analyzed the capacity of the three-node network consisting of a source, a destination, and a relay. It was assumed that all nodes operate in the same band, so the system can be decomposed into a broadcast channel from the viewpoint of the source and a multiple access channel from the viewpoint of the destination (Fig. 3). Many ideas that appeared later in the cooperation literature were first exposited in [16].

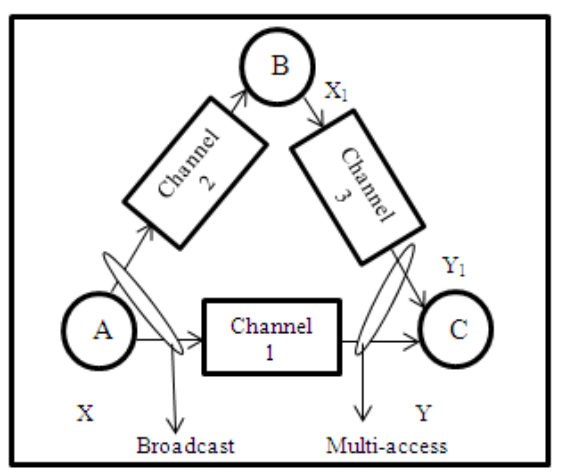

Fig. 3. The relay channel

However, in many respects the cooperative communication we consider is different from the relay channel. First, recent developments are motivated by the concept of diversity in a fading channel, while Cover and El Gamal mostly analyze capacity in an additive white Gaussian noise (AWGN) channel. Second, in the relay channel, the relay's sole purpose is to help the main channel, whereas in cooperation the total system resources are fixed, and users act both as information sources as well as relays. Therefore, although the historical importance of [16] is indisputable, recent work in cooperation has taken a somewhat different emphasis [2].

\subsection{Benefits of cooperative transmission}

From the perspective of the network, cooperation can benefit not only the nodes involved, but the whole network in many different aspects. For illustration purposes, we choose to explain only a few potential benefits below [20].

(1) Higher spatial diversity
In this example, Fig. 4 shows a small network of four nodes. If the channel quality between nodes $\mathrm{S}$ and $\mathrm{D}$ degrades severely (e.g., due to shadow or small-scale fading), a direct transmission between these two nodes may have an intolerable error rate, which in turn leads to retransmissions. Alternatively, S can use spatial diversity by having a relay $\mathrm{R} 1$ overhear the transmissions and then forward the packet to D as discussed above. The source $S$ may resort to yet another terminal R2 for help in forwarding the information, or use R1 and R2 concurrently. Similar ideas apply to larger networks as well. Therefore, compared with direct transmission, the cooperative approach enjoys a higher successful transmission probability. We note here that cooperative communications has the ability to adapt and to mitigate the effects of shadow fading better than MIMO since, unlike MIMO, antenna elements of a cooperative virtual antenna array are separated in space and experience different shadow fading.

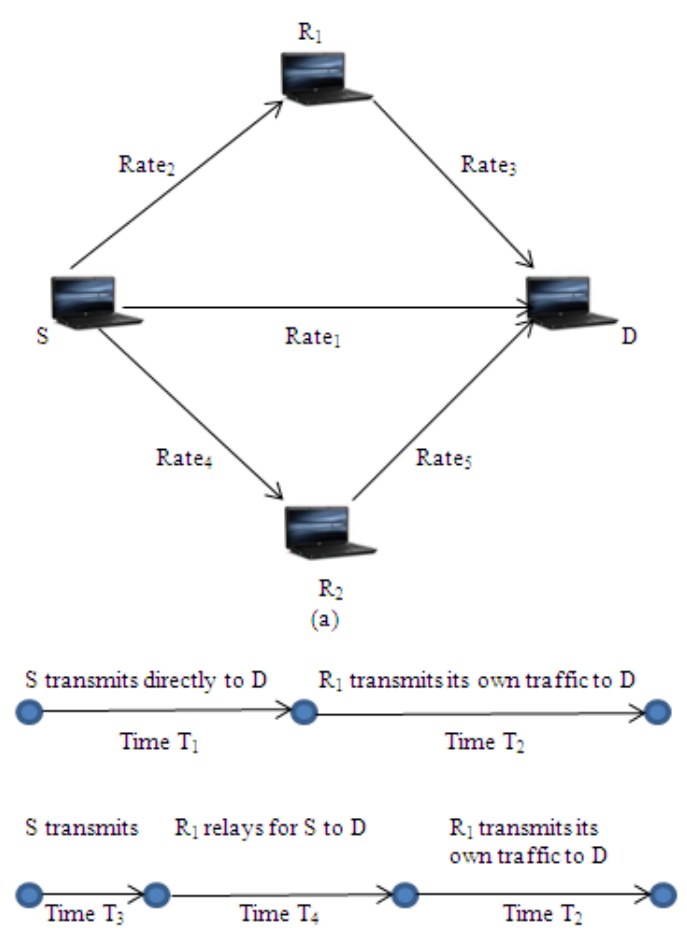

(b)

Fig. 4. a) Cooperation in a network; b) illustration of the delay and throughput improvement achieved by cooperation in the time domain

\section{(2) Higher throughput-lower delay}

At the physical layer, rate adaptation is achieved through adaptive modulation and adaptive channel coding. Many MAC protocols have introduced rate adaptation to combat adverse channel conditions. For instance, when a high channel error rate is encountered due to a low average SNR, the wireless LAN standard IEEE 802.11 switches to a lower transmission rate so as to guarantee a certain error rate. The power of cooperation is evident when it is applied in conjunction with any rate adaptation algorithm. In Fig. 4a, specifically, if Rate 2 and Rate 3 are higher than Rate1 such that the total transmission time for the two-hop case through R2 is smaller than that of the 
direct transmission, cooperation readily outperforms the legacy direct transmission, in terms of both throughput and delay perceived by the source S. Furthermore, for relays such as $\mathrm{R} 1$ and $\mathrm{R} 2$, it turns out that their own individual self-interest can be best served by helping others. As further illustrated in Fig. 4b, the intermediate node R1 that cooperates enjoys the benefit of lower channel-access delay, which in turn can be translated into higher throughput. It is worthwhile to note that Fig. 4b also draws a rough analogy with the cooperative scheme discussed above and illustrates that rate adaptation can further improve the benefits of cooperation in a network setting.

\section{(3) Lower power consumption and lower interference}

The diversity, error rate, and throughput gains obtained through cooperation can be traded in for power savings at the terminals. Alternatively, cooperation leads to an extended coverage area when the performance metric (error rate, throughput, etc.) is fixed. The advantage of cooperation also leads to reduced interference when the network is deployed in a cellular fashion to reuse a limited bandwidth. With the improvement of throughput, we can reduce the average channel time used by each station to transfer a certain amount of traffic over the network. Therefore, the signal- to-interference ratio (SIR) between proximal cells using the same channel can be reduced, and a more uniform coverage can be achieved. As wireless network deployments become ever denser, a reduction of SIR will directly lead to a boost in network capacity. Indeed, the problem of dense deployment has already been reported for IEEE 802.11 b/g networks, which have only three no overlapping channels.

\section{(4) Adaptability to network conditions}

The cooperative communication paradigm allows wireless terminals to seamlessly adapt to changing channel and interference conditions. The choice of relays, cooperation strategy, and the amount of resources available for cooperation can be opportunistically decided. For example, in Fig. $4 \mathrm{a}$, if the source $\mathrm{S}$ has some information about the current channel gains, packet-loss rates, traffic conditions, interference, or remaining battery energy of nodes in the network, it may choose to transmit its information directly to its destination $\mathrm{D}$, using $\mathrm{R} 1$ or $\mathrm{R} 2$ or both in a cooperative fashion, depending on which transmission mode results in better performance (in terms of error rates, throughput, or power). This way, a surplus of resources such as battery energy or bandwidth at a particular node can be utilized by other nodes in the network in a manner that will benefit everyone, including the relay node itself. Although originating from physicallayer cooperation, all the aforementioned benefits cannot be fully realized until proper mechanisms have been incorporated at higher protocol layers (e.g., MAC, network) and the necessary information is made available from the lower layer (e.g., PHY). Indeed, a cross-layer approach has to be followed to reap all the benefits of cooperation. As we illustrate via the cooperative MAC protocol described in the following section, an additional three-way handshake procedure and a new signaling message have to be introduced to the MAC layer, and information on channel conditions for related wireless links should be made available to the upper layers so that the cooperation can be fully enabled. Another example of a cross-layer approach to cooperation, which involves interaction between the application layer and the physical layer, is provided in form transmission of video signals over wireless links.

\section{(5) When a packet is not received at the destination}

Normally, in ARQ protocols, when a packet is not received at the Destination (or received with error), the following happens: (i)The packet is lost (discarded), (ii)An ACK is NOT generated from the Destination, (iii)The Source will retransmit the packet later.

With Cooperative Communication, when a packet is not received at the Destination (or received with error), the following happens: (i)The packet is lost (discarded), (ii)An ACK is NOT generated by the Destination, (iii)A relay node that successfully overheard the packet may relay this to the Destination, (iv)The Source will retransmit if there no successful relay.

\section{COOPERATIVE Routing IN WIRELESS Mesh NETWORKS}

A wireless mesh network composed of heterogeneous nodes, ranging from energy constrained mobile devices to high performance fixed nodes [21]. The convergence of heterogeneous wireless networks is a movement in the growth of wireless networks. A new architecture for network convergence, named Wireless Cooperative Mesh Network, is proposed to explain such rising problems in convergence like transmission mode selection, load balancing, routing and handover. The new architecture is based on the structure of Wireless Mesh Networks (WMNs), and cooperative communication is also employed to optimize its structure and improve its dedication. It can therefore get hold of compensation of both the Mesh technology (high spectrum efficiency and dynamic self-organization) and cooperative communication (high diversity gain and high energy efficiency). Although the merits of cooperative relaying in a small isolated wireless network composed of one single source-relay destination hop has been explored sufficiently, there are still many challenges when cooperative diversity is to be used in large-scale wireless mesh networks [21].

\subsection{Overview of Cooperative Mesh Network}

Mesh structure and cooperative communications are employed to further optimize the structure and upgrade the performance. The architecture is shown in Fig. 5. First of all, the wireless Mesh backbone is formed by Mesh routers, and then the wireless Mesh user network is formed by users. Users have to access the wireless Mesh backbone to get service. Users close to the Mesh routers are connected directly to the wireless Mesh backbone, while those, which are far away from the Mesh routers, are connected to the wireless Mesh backbone through multi-hop. 


\subsection{Advantages of wireless cooperative mesh network}

The foremost advantages of wireless cooperative mesh network include the following:

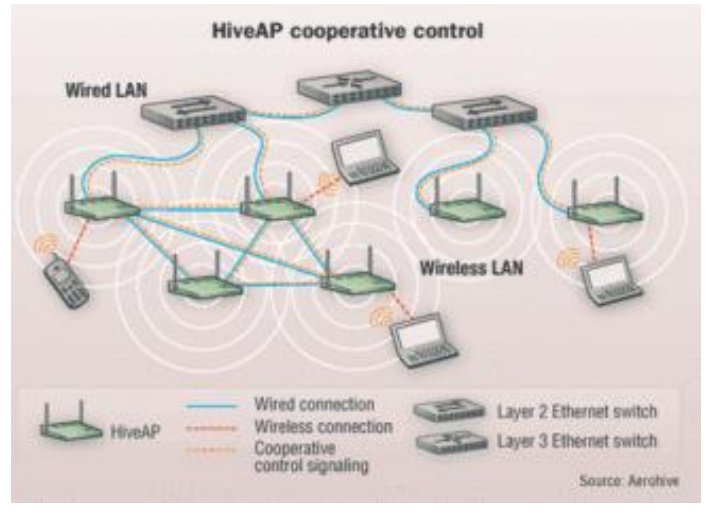

Fig. 5. Wireless Cooperative Mesh Network

(1) Load balancing: Load balancing is one of the major problems in convergent networks. Nodes near the centre of the network bear more forwarding services than those near the margin of the network [22]. For this reason the nodes near the centre of the network to use up their energy very fast and make the node density around the centre lower, that will therefore cause the load of other nodes in this area to turn into heavier and form a vicious circle. This kind of regional reduction of node density will not only result in the partition of the network, but also affect the services in progress and reduce the network throughput. Therefore, the problem of load balancing in convergent networks requires in-depth study. In the Wireless Cooperative Mesh Network there are multiple backup paths, instead of only one path, are searched [22].

(2) Routing: In cooperative mesh network, there are infrastructures laid beforehand, as well as some fixed relay nodes which are more capable in computing and storage [22]. These relay nodes, that have full or partial purpose of a BS, can gather the location information of nodes and the topology information of the network surrounded by certain coverage region, and can thus assist the computing of routing and the selection of paths. Therefore, in the design of the routing mechanism of convergent networks, these nodes should be made full use of [22]. In this way, the distributed routing is combined with the relay-assisted routing to enhance the network performance.

(3) Handover: For instance, group handover necessities are produced at the time trains, buses or other vehicles are on the way. However rising residual capacity can resolve the problem, this will reason why a waste of network resources and boost the call blocking rate. Cooperative handover can be employed to solve the problem. The basic idea of cooperative handover is to balance the load through the utilization of cooperative communications. Conventional handover approach is come across with a problem to choose between received signal strength and cell blocking rate. In the Wireless Cooperative Mesh Network, the only criterion of handover is to access the adjacent cell which bears the lightest load, and cooperative multi-hop is employed to ensure the reliability of handover. The MS initiates the handover requirement, and then the cell, which bears the lightest load, computes the routing information and determines the path for the MS (i.e., designates relays to provide routing for the MS) according to the connection status of the network and at the meanwhile allocates corresponding bandwidth [22]. As well, wireless routers can be permanent on vehicles to serve as the handover requisite agent for set of services.

\subsection{Applications}

The paper [23] presents the Eurecom testbed OpenAirMesh, which is an experimental real-time hardware and software platform for cooperative mesh networks. This effort can be seen as a mock standard for realistic experimentation purposes which retains the salient features of a real radio system, without all the required mechanisms one would find in a standard used in deployment of commercial networks. One major application of OpenAirMesh platform is the demonstration of rapidly-deployable broadband ad hoc communications systems for public safety units in interventions natural hazards and industrial accidents.

\subsection{State-of-the-art routing strategies in cooperative $W M N$}

Liping Wang et al. [21] model and estimate two cGeorouting schemes which is Cooperative- Random Progress Forwarding (C-RPF) and Cooperative-Nearest with Forward Progress (C-NFP).cGeo-routing significantly increases the average transport capacity for a single hop in well-connected mesh networks, and the gain increases with the transmitted signal-to noise ratio (SNR).

In geographic routing, each node selects the next-hop node based on its own position and the positions of its neighbors and the destination. Finding a route in such a hop-by-hop way avoids high routing overhead and therefore achieves scalability. It is one of the major reasons for using this type of network [21]. In [21], authors focus on cooperative geographic routing (cGeorouting). The integration of cooperative transmission and geographic routing is to exploit cooperative diversity in mesh networks.

To gain cooperative diversity in wireless mesh networks the traditional Geo-routing included and proposed a cooperative geographic routing (cGeo-routing) strategy, which allows cooperative relaying in each hop. With this strategy, the sender S currently holding the packet selects one of its neighbors as a cooperative relay $\mathrm{R}$, and then $\mathrm{R}$ selects one of its neighbors as a receiver $\mathrm{V}$. The transmission in every sender relay- receiver (S-R-V) hop follows a selective decode-and forward (DF) relaying scheme. If direct transmission from $\mathrm{S}$ to $\mathrm{V}$ achieves higher data rate, $\mathrm{S}$ sends the packet directly to $\mathrm{V}$. Otherwise, DF cooperative relaying among SR- V is performed. Finally, V combines the signals received respectively from $S$ and $R$ to decode the packet.

cGeo-routing gains lower single-hop throughput than its corresponding Geo-routing scheme. The average 
transport capacity of a cGeo-routing scheme is significantly higher than that of its corresponding Georouting scheme.

Christian Ibars et al. [24] describe two cooperative strategies are proposed: opportunistic relaying, and partial decoding. Authors proposed a method to integrate cooperative techniques in wireless mesh networks in order to increase their throughput. Mainly, two cooperative strategies are proposed: opportunistic relaying, and partial decoding

In the proposed system, cooperative techniques are used to establish virtual links among nodes in the wireless backhaul. The purpose of virtual links is to hide cooperative strategies from the network layer, so that traditional (noncooperative) routing protocols can be used. Inside a virtual link, which is distinct by the carrier sensing and connectivity regions, nodes cooperate using opportunistic relaying or partial decoding. The end-to-end performance of the cooperative backhaul was evaluated in terms of throughput and outage probability [24]. A wireless backhaul with a linear topology was estimate, in cooperation for line of sight and non-line of sight propagation.

The study is based on cross-layer problem of combining routing and cooperative diversity in multi-hop, bandwidth constrained, networks with dedicated multiple access. Cooperative diversity is implemented using Selection Cooperation. However, if cooperative diversity is incorporated into the route selection algorithm, the number of required hops decreases and significant gains are possible [23]. A natural and also simple, selective implementation of cooperative diversity into one-hop systems has analogous performance to the further complex routing algorithms. Including the search for cooperative nodes into the dynamic route search, however, does further increase flow rates by decreasing the average number of hops and thus decreasing the required bandwidth expansion [25]. Here Dynamic Cooperative Routing is used. Dynamic Cooperative Routing simultaneously combines Dynamic Routing with Cooperation: the optimal path is chosen together with the cooperative partners. Because of Dynamic Routing, the Dynamic Cooperative Rate $\mathrm{R}^{\mathrm{DCR}}$ is the maximum of the rates achieved with different hops:

$$
\mathrm{R}^{\mathrm{DCR}}=\max \left\{\mathrm{R}^{1} ; \mathrm{R}^{2} ;::: ; \mathrm{R}^{\mathrm{M}+1}\right\}
$$

Distinguishing Dynamic Routing, however, $\mathrm{R} D C R$ is achieved with Cooperation potentially included in each hops, i.e., for every possible permutation of hops, the algorithm outfit Smart Cooperation along each hop.

In [26] a new cooperative communications method (CCM) for the IEEE802.16 mesh mode. CCM devise a novel algorithm for deciding on cooperation nodes, which look upon as both channel state of physical layer and overhead on MAC layer. It also adopts a new scheme to schedule minislots and coordinate the transmission from the source node to cooperation nodes and the transmission from cooperation nodes to the destination node. CCM can select optimal cooperation nodes according to dynamic network scenarios, and in turn increase the channel capacity and improve the system performance.

\section{COOPERATIVE ROUTING STRATEGIES IN AD HOC NETWORKS}

In a mobile ad hoc network, mobile nodes are dynamically and arbitrarily located and they communicate with each other through one or more relaying mobile terminals [27].

A Routing protocol is used to discover routes between nodes. The primary goal of an Ad hoc network routing protocol is to establish a correct and efficient route between a pair of nodes so that messages could be delivered properly. Route construction should be done with a minimum of overhead and bandwidth consumption. In [28], the authors provided an overview of eight different protocols by presenting their characteristics and functionality, and then gave a comparison and discussion of their respective merits and drawbacks.

In [29], several multihop routing schemes, such as 'selection based on Pathloss' and 'selection based on minimal transmission power', were proposed and evaluated. In those strategies certain mobile terminals that have good communication links with the base station were used to act as relay nodes for those that do not have. Relaying via wireless terminals can have a significant impact on transmission power, SIR, and the proportion of content. This is mainly due to the fact that the signals only have to travel through shorter distances and/or improved line-of-sight paths.

On the other hand, cooperative diversity has become more and more attractive recently since it could mitigate the particularly severe channel impairments arising from multipath propagation [30], where sets of terminals relay signals for each other to create a virtual antenna array, trading off the costs-in power, bandwidth, and complexity-for the greater benefits gained by exploiting spatial diversity in the channel. By contrast, classical network architectures only employ point-to- point transmission and thus forego these benefits [17].

Therefore, cooperative diversity can provide full spatial diversity, as if each terminal had as many transmit antennas as the entire set of cooperating terminals. Such diversity gains translate into greatly improved robustness to fading for the same transmit power, or substantially reduced transmission power for the same level of performance [31].

\subsection{Overview of Cooperative Ad hoc Network}

Ad hoc wireless networks consist of wireless nodes that can communicate with each other in the absence of a fixed infrastructure [32]. Ad hoc networks, defined in a manner in which the network nodes are organized to provide pathways for data to be routed from the user to and from the desired destination. Fortunately, cooperative communication, a new paradigm for wireless communication, has emerged, where wireless nodes cooperate with each other in their transmissions to form a longer transmission link [33]. Meanwhile, as a new 
research area, cooperative transmission has attracted much attention as an effective technique to combat multipath fading, enhance receiver reliability and achieve better energy efficiency of wireless communication systems in ad hoc network.

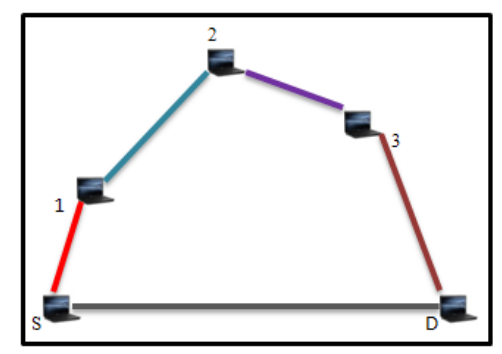

Fig. 6. Multihop Routing

This is the simple cooperative routing example. Suppose there are 5-node wireless ad hoc network, where $\mathrm{S}$ is the source and $\mathrm{D}$ is destination nodes, respectively. In Fig. 6 shows multihop routing, where information is transferred from one node to another until target is achieved.

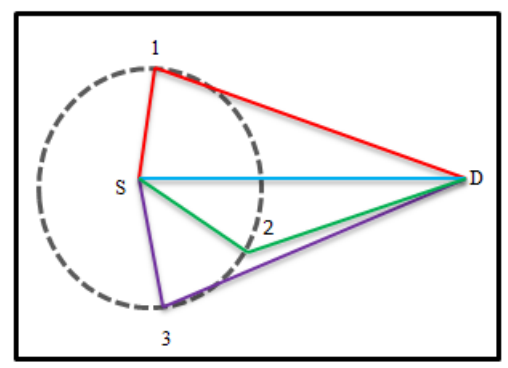

Fig. 7. Cooperative Routing

Suppose we can go through optimal multi hop path based on some routing scheme from $\mathrm{S}$ to $\mathrm{D}$ is through node 1 . At the same time, node 2 and 3 , which are also located within the transmission radius of $S$ to 1 , receive the information transmitted from $\mathrm{S}$ at no additional cost. Then in the second step, cooperation between node 1,2 and 3 will form transmission side diversity, which will consume lower power. Here it is assumed that each node can participate in cooperative transmission after it has completely received the information.

\subsection{Advantages of Cooperative Ad Hoc Networks}

The principal advantages of a cooperative Ad hoc network include the following:

(1) They do not require infrastructure and promise greater flexibility, lower operating cost, higher throughput and better coverage [31].

(2) More recently, it is shown that cooperative communication can provide increase capacity and power savings in ad-hoc networks [34].

(3) In cooperative wireless networks, the nodes may help each other by opportunistic overhearing, combining and error correcting the source packets prior to forwarding. The operation of store-and-forward can be executed through medium access control (MAC) protocols [35].
(4) In most wireless Ad hoc networks, the nodes compete for access to shared wireless medium, often resulting in collisions (interference). Using cooperative wireless communications improves immunity to interference by having the destination node combine selfinterference and other-node interference to improve decoding of the desired signal.

(5) The transmission time can be less in cooperative communication.

(6) Cooperative Ad hoc network provide Cooperative diversity. Cooperative diversity is a cooperative multiple antenna technique for improving or maximizing total network channel capacities for any given set of bandwidths which exploits user diversity by decoding the combined signal of the relayed signal and the direct signal in wireless multi hop networks.

(7) Cooperative path routing has two major benefits. First, cooperative path routing can gain higher energy saving than non-cooperative shortest path routing. Our empirical results point to that with more nodes added in the network, more energy saving can be achieved by cooperative routing since a dense network offers more opportunities for cooperative transmissions. Second, cooperative transmission greatly alleviates the scalability problem in wireless networks [36].

\subsection{Key Applications of Cooperative Ad hoc Network}

Cooperative Ad hoc network sustain cooperative Caching. Cooperative caching, which allows the sharing and coordination of cached data among multiple nodes, can further explore the potential of the caching techniques [37]. Caching techniques use to efficiently support data access in ad hoc networks. For example they use in mainly three scheme: CachePath, CacheData, and HybridCache. In CacheData, intermediate nodes cache the data to serve future requests instead of fetching data from the data center. In CachePath, mobile nodes cache the data path and use it to redirect future requests to the nearby node which has the data instead of the faraway data center. HybridCache takes advantage of CacheData and CachePath while avoiding their weaknesses

Vehicular Ad-Hoc networks (VANETs) are playing a critical role in enabling important active safety applications such as collision warning and vehicle tracking. The most pressing challenge in enabling such applications is to maximize the amount of disseminated vehicle state information while avoiding network congestion. The most challenging application planned for deployment over VANETs is Cooperative Active Safety (CAS) using cooperative communication. In the CAS concept, vehicles will send self-information, e.g. GPS position, speed, and heading, to neighboring vehicles over a wireless channel. The receiving vehicle can use the incoming messages to track the sending vehicle and detect if there is a threat. If so, it may warn its driver or perform emergent reactions [38].

\subsection{State-of-the-art routing strategies in cooperative $\mathrm{Ad}$ Hoc Networks}

In [31] cooperative diversity included in route selection, both power based and physical distance-based strategies 
could save power rather than simple multi-hop network structure. Besides this, we can say that the more nodes are permitted to take part in cooperation; the more energy could be saved. In [31], authors assume, each terminal has a single omni-directional antenna and multiple nodes are allowed to transmit their messages simultaneously. And also, each node can dynamically adjust its transmitted power and phase to control its transmission range and possibly synchronize with other nodes. There proposed metrics are based on power consumption and also physical distance.

It is assumed that the received information can be decoded without error if the received $S N R$ level is above a minimum threshold min $S N R$ and that no information is received otherwise. They derived link cost between $S$ and D. Based on this they conclude that compared with traditional direct transmission, cooperative routing scheme can save more power than simple multihop strategy. And the reason is that through cooperative diversity, sets of wireless terminals benefit by relaying messages for each other to propagate redundant signals over multiple paths in the network [30]. This redundancy allows the ultimate receivers to essentially average channel variations resulting from fading, shadowing, and other forms of interference [31]. The more nodes participate in cooperation, the more energy could be saved.

In [50], the authors propose a Capacity-Optimized COoperative (COCO) topology control scheme to improve the network capacity in mobile ad hoc networks (MANETs) by jointly considering both upper layer network capacity and physical layer cooperative communications. The network topology in a MANET is changing dynamically due to user mobility, traffic, node batteries, and so on. Meanwhile, the topology in a MANET is controllable by adjusting some parameters such as the transmission power, channel assignment, etc. As topology control is to determine the existence of wireless links subject to network connectivity, the general topology control problem can be expressed as

$$
\mathrm{G}^{*}=\arg \max f(\mathrm{G}),
$$

\section{s.t. network connectivity.}

The problem Eq. 2 uses the original network topology $\mathrm{G}$, which contains mobile nodes and link connections, as the input. According to the objective function, a better topology $\mathrm{G}^{*}\left(\mathrm{~V}, \mathrm{E}^{*}\right)$ will be constructed as the output of the algorithm. $\mathrm{G}^{*}$ should contain all mobile nodes in $\mathrm{G}$, and the link connections $\mathrm{E}^{*}$ should preserve network connectivity without partitioning the network. The structure of resulting topology is strongly related to the optimization objective function, which is $f(\mathrm{G})$ in Eq. 1. It is difficult to collect the entire network information in MANETs. Therefore, it is desirable to design a distributed algorithm, which generally requires only local knowledge, and the algorithm is run at every node independently. Consequently, each node in the network is responsible for managing the links to all its neighbors only. If all the neighbor connections are preserved, the end-to-end connectivity is then guaranteed. Given a neighborhood graph GN (VN, EN) with $N$ neighboring nodes, we can define a distributed topology control problem as $\mathrm{G}^{*} N=\arg \max f(\mathrm{G} N)$, s.t. connectivity to all the neighbors. The objective function $f(\mathrm{G})$ in Eq. 1 is critical to topology control problems. Network capacity is an important objective function. [50]

Nam-Soo Kim [34] introduced Cooperative Diversitybased Routing (CDR) Scheme which exploit the cooperative space diversity for power saving and for performance enhancement of wireless ad-hoc networks. Proposed CDR is given below:

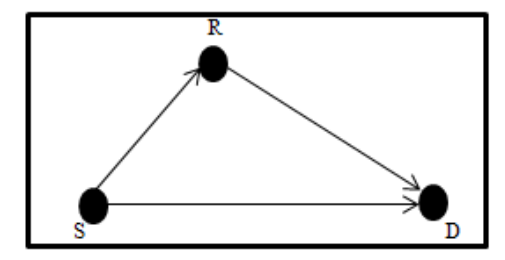

Fig. 8. Simple Cooperative Diversity Model

They describe a model of a cooperative diversity, which has single relay node, is shown in Fig. 8. Where $S$ is source node, $\mathrm{R}$ is relay node, and $\mathrm{D}$ is destination node, respectively. At first the transmission from the source node is started. Then, once the message is received at both of the receivers, the relay node regenerates and transmits the message at a later time slot. The destination will compare the signals from both the source and the relay node separately, and selects the signal with higher instantaneous SNR for decision to utilize the selection combining diversity [34].

They calculate outage probability and the probability that the message is transmitted successfully from one node to another, respectively. The required average SNR for the target outage probability increases with the number of nodes in MMR. However, the required average SNR decreases with the increase of the number of nodes in CDR [34] .By increasing number of nodes in CDR causes target outage probability is reduced. The limitation of the paper is that for better transmitting power they sacrifices throughput.

Considering new challenge in cooperative wireless networks, Shoukang Zheng proposed in a routing metric ETTC (Expected Transmission Time with Cooperation) based on average transmission time with the opportunistic cooperation. Their goal is to calculate optimum route and select the best next hop with a MAC layer based on cooperative 802.11 MAC. Node $s$ looks into its routing table to find its next hop $d$.From this figure we can see that cooperative approach helps reducing the transmission time to the next hop. The transmission time can be less in spite of relaying requires an additional transmission, because relay nodes $r$ which are within the range of both $s$ and $d$ have a small link distance compared to the link between node $s$ and node $d$. The cooperation gain is achieved due to transmission time reduction.

In Fig. 9 the path metric assigned to the graph is the transmission time. We have to reach destination node $v 6$ through source node is $v 1 . v 4$ has the potential cooperative node $v 3$ to help transmit. Although hop-count 
based routing protocol prefers the route $v 1 \rightarrow v 2 \rightarrow v 6$ for the transmission from $v 1$ to $v 6$, the routing metric is based on of transmission time that determines the route $v 1 \rightarrow v 4 \rightarrow v 5 \rightarrow v 6$ for the flow from $v 1$ to $v 6$.we can see that minimum hop count based scheme has less hops than the proposed scheme.

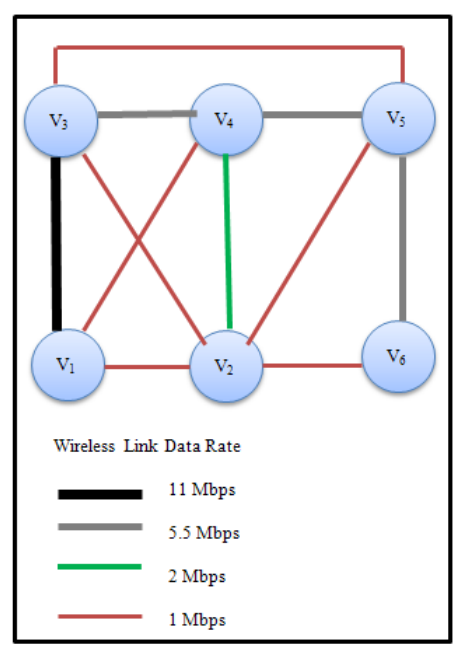

Fig. 9. Network topology

Since the selection of cooperative nodes could be decided at random and the route is updated in a greater timescale compared with the change of the channel states, a statistical estimation of average transmission time among the neighboring nodes is more reasonable [35].

In this paper cooperation gain is measure using approximation of time spend between two node. The routing metric ETTC $(\mathrm{s}, \mathrm{d})$ between parent node $s$ and its candidate next hop node $d$ as follows:

$$
\operatorname{ETTC}(\mathrm{s}, \mathrm{d})=\operatorname{Tsrd} \mathrm{Pc}+\operatorname{Tsd}(1-\mathrm{Pc})
$$

Where $P c$ is the percentage of time spent on the cooperative transmissions for all the helper nodes, Tsrd is the average transmission time per packet with cooperation, and Tsd is the average transmission time per packet without cooperation.

ETTC scheme help in exploiting the cooperation gain effectively in terms of end-to-end delay and throughput. In this paper Zigui Yang investigates the power saving that can be achieved by using advanced relay-channel signaling rather than traditional multi-hop routing. In this paper, authors consider a "connection oriented" unicast problem without carrier synchronization when the source needs to continuously send a sequence of messages over one fixed route [39]. Author use decode forward scheme as it is regarded as more practical in large networks.

They prove two theorems stating Theorem 1 shows that if we find a best sequential relaying path, it is guaranteed to be optimal. Theorem 2: For an optimal sequential relaying path, the optimal power allocation policy can be implemented by a recursive power filling procedure, i.e., starting from the source; each node adjusts its transmission power such that condition is satisfied with equality sign for its immediate child [39].
In [39] authors proposed two algorithms one is CTNCR. In this heuristics, we first find a shortest non-cooperative path using the standard Bellman- Ford algorithm based on link-based metric and then use power filling procedure to determine the overall power consumption. Other algorithm is SNER which is essentially a greedy algorithm similar to the Prim-Dijkstra spanning tree algorithm but it stops whenever the destination is included in the tree.

Cooperative routing yields large power savings in low attenuation regimes compared to the traditional multi-hop operation and the gains are mainly due to more advanced signal processing than routing[39].

In this paper, Min Sheng described a routing protocol SCR (Small world based Cooperative Routing protocol) for large scale wireless ad hoc networks are developed with combination of small world and cooperative communications.

In small world average path length is short. If we can introduce the small world phenomenon to large scale wireless ad hoc networks, the path length between every pair of nodes may be decreased distinctly [33]. We know, multi-hop wireless ad hoc networks can only be described as a SG (Spatial Graph), where the links are determined by the radio connectivity. The most fundamental distinction between $R G$ and $S G$ is that one link in $R G$ may be several hops away as in SG. SCR protocol mainly focus on the following: 1) Short-cut node selection based on small world phenomenon; 2) End-toend path establishment based on greedy routing algorithm; 3) Packet forwarding strategy by using cooperative communication link

The reason for selecting short-cut nodes is that corresponding cooperative short-cut links can reduce the average path length significantly in a network. Here endto-end path will be recognized based on greed routing algorithm. However the SCR regards the short-cut node is selected based on cooperative shut-cut link.

If the probability that the length of the cooperative short-cut link is longer that the distance between the forwarding node and its short-cut node is high, the small world phenomenon in a large scale wireless network can be restored approximately [33]. The path length will be decrease a lot. From this paper author shows that the average path length of SCR is decreased noticeably with the number of cooperation partners increasing. And also, the shorter the average length of a path, and the more efficiency of the SCR cooperative routing there is a comparison between SCR with AODV and DSR routing protocols by discussing the overheads and the packet loss rate. Overhead and packet loss is less in SCR.

\section{COOPERATIVE ROUTING IN WIRELESS SENSOR NETWORKS}

Wireless sensor networks (WSNs) are often deployed to perform a specific set of tasks. Therefore, the design of such a network should be optimized for these tasks. Consider, for example, a network of distributed sensors for the detection of certain events or phenomena. Sensors 
may have a limited communications range, and their local detection may be unreliable. Thus, it is necessary to aggregate the data collected from multiple sensors and send them to a fusion center where a global decision can be made. The measurements at individual sensors, however, are often spatially correlated, and delivering all of this raw data to the fusion center may be both inefficient (in utilization of the network's resources) and unnecessary (for accurate detection). It is in this context that cooperative networking is especially appealing for sensor networks [40].

\subsection{Overview of Cooperative sensor network}

Promising wireless applications such as sensor and wireless mesh networks have a growing demand for small and low cost devices that are densely deployed over a wide area. The inadequate battery life span of devices and the scarce bandwidth shared by a large amount of users often obstruct the development of these systems. Therefore, many research efforts have been made to maximize the system performance under the respective resource constraints. However, the effectiveness of these solutions could be limited by the uneven resource distribution or the diverse channel quality among users, which is especially true in highly dynamic and/or hostile environments [41]. Fascinatingly, some of these issues can be improved or resolved if users are prepared to share their local resources and cooperate in transmitting each other's messages which is the spirit of cooperative communications.

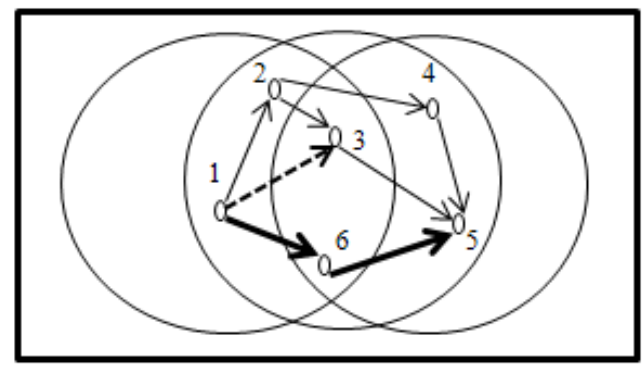

Fig. 10. Relay paths with equivalent or remedy nodes

From Xiaoxia Huang [42] paper we get the architecture of cooperative sensor network. As nearby nodes with a copy serve as caches, the next-hop node could retrieve the packet from any of them. Suppose node 1 attempts to deliver a packet to node 5 over path $1-3-5$. When 1 transmits to node 3 , nodes 2 and 6 may also correctly receive the packet. Cooperation among those nodes may result in high energy-efficiency and robustness when we carefully utilize diversity. If link 1-3 fails due to deep fading or the departure of node 3 , then node 3 cannot receive the packet correctly. Without waiting for potential multiple retransmissions over the unreliable or disappeared link $1-3$ before re-routing or dropping the packet, a substitute link 2-4 or $6-5$ could transfer the packet proactively. As long as at least one link is capable of delivering the packet successfully, the packet can be received and further forwarded towards the destination. In [42] authors assume the wireless sensor network is densely deployed, so each node has plenty of neighbors.
In wireless networks, path breakage occurs more frequently due to channel fading, shadowing, interference, node mobility as well as power failure. When a path breaks, rerouting or alternative routing may be necessary and should be carried out promptly dense wireless sensor networks offer the opportunity to develop novel communication and routing techniques based on cooperation among nodes in the neighborhood. The failure probability of all links is much smaller than that of a single link [42].

\subsection{Advantages of Cooperative Sensor Network}

The main advantages of cooperative wireless sensor network include the following:

(1) In wireless sensor networks, path breakage occurs frequently due to node mobility, node failure, and channel impairments. Cooperative sensor network combat path breakage with minimal control overhead. In Wireless Broadcast Advantage (WBA), all nodes inside the transmission range of a single transmitting node may receive the packet; hence naturally they can serve as cooperative caching and backup nodes if the intended receiver fails to receive the packet [42].

(2) In wireless sensor networks, the radio interference and the multipath fading make wireless transmission unreliable. The cooperative delivery diversity method is an effective approach to combating multipath fading [43].

(3) Cooperative diversity is a strategy proven to offer significant gain to combat fading in wireless networks. This strategy can be applied in Wireless Sensor Networks (WSN) to offer a significant increase to the network lifetime [44].

(4) EnviroStore, is a cooperative storage system that maximizes network storage capacity. EnviroStore can (i) effectively utilize the network storage capacity of disconnected sensor networks to accommodate the most sensory data, and (ii) opportunistically offload data from overloaded network partitions to underloaded partitions via mules [45].

\subsection{Key Applications of Cooperative Sensor Network}

A significant number of applications, however, do not require real-time information. They collect information in a month or specific period of time. There is no longer a need to uphold a base station. A user no longer has to worry about powering up the base station in the wilderness, harsh weather and animals, and enduring the risk of losing data because of a centralized point of failure.

The goal of the system is to maximize its data storage capacity by appropriately distributing storage utilization and opportunistically offloading data to external devices when possible [45]. The observation is that a large category of sensor network applications, such as environmental data logging, does not require real-time data access. Such networks generally operate in a disconnected mode. Rather than focusing on multi hop routing to a base station, an important concern becomes (i) to maximize the effective storage capacity of the disconnected sensor network such that it accommodates the most data, and (ii) to take the best advantage of data 
upload opportunities when they become available to relieve network storage [61]. The storage system described in this paper achieves the above goals, leading to significant improvements in the amount of data collected compared to non-cooperative. EnviroStore takes into account the rate of energy consumption to avoid depletion related data loss rate [45].

Cooperative sensor network create advancement in traffic system also. The advancement of information and communication technologies accelerates the development and deployment of intelligent transportation systems. In the future cooperative traffic system, actors collect information about their immediate environment sharing it with each other, enabling all traffic entities to become context-aware.

\subsection{State-of-the-art routing strategies in Cooperative Sensor Network}

In [51], the authors study the problem of how to strike a balance between the QoS provisioning and the energy efficiency when a cooperative communication scheme is applied to a clustered wireless sensor network. Xiaoxia Huang et al. [42] present a distributed robust routing protocol in which nodes work cooperatively to enhance the robustness of routing against path breakage. This paper describes a cross-layer robust routing protocol based on node cooperation among nearby nodes for unreliable mobile wireless sensor networks. Inside the robust path expanded from an intended path, a reliable path is selected for packet delivery. Depend on the path quality; the intended path is able to look after the varying topology. Utilizing path diversity in the robust path, the robust routing protocol is capable of selecting the best path in a wide zone for each packet. This is the difference of our RRP from traditional routing protocols. Therefore, the robustness against path breakage is improved [42].

This paper exploits the idea of cross-layer design for wireless sensor networks to improve the network performance. The paper presents a new energy efficient cooperative routing scheme with space diversity using space-time block codes (STBCs) at the same time as improving the link's energy-efficiency. In our solution, the selected multiple nodes act as multiple transmitting and receiving antennas. Full diversity from the orthogonal STBC is utilized to overcome multipath fading and to enhance power efficiency. The network performance measures, such as, network throughput and delay are analyzed via an M/G/1 queuing model [43]. The energy consumption and protocol efficiency explored.

Node cooperation is one of the unique aspect differentiating wireless sensor networks (WSNs) from usual wireless cellular networks. This paper give focus on joint clustering and optimal cooperative routing, where neighboring nodes dynamically form coalitions and cooperatively transmit packets to the next hop destination. We show that the cooperative sensor network can be modeled as an edge-weighted graph, based on which minimum energy cooperative routing is characterized by using the standard shortest path algorithm [44]. They give attention on energy-delay-constrained maximum throughput routing, which is known to be NP-hard.
Mainly look after in two individual cases firstly, where the delay can be expressed in terms of the number of hops, by using the bi-section method to find the maximum throughput routing; secondly For large scale networks where the end-to-end delay can be approximated as the product of the number of hops and the average one-hop delay, we present a polynomial time algorithm to find the maximum throughput routing. Here well-known Dijkstra's algorithm is used to find the minimum energy cooperative routing path. The minimum energy cooperative routing would achieve higher energy saving than cooperative geographic routing, simply because it makes use of optimal clustering to minimize the transmission cost and chooses the optimal routing path based on global information [44].

In [46], the novel node selection technique is proposed in cooperative wireless networks. The usage of proposed technique in single-hop network transmission with multiple relays between the source and destination will produce better results comparing to the existing techniques such as distributed space-time codes. This paper suggests a node selection idea in cooperative wireless networks. In a single-hop network with multiple relays, selecting a single node to aid in the transmission between a source and a destination outperforms both traditional orthogonal transmissions and distributed space-time codes. The usage of multiple hops will better the performance of wireless communication. In order to further improve the performance in wireless communication, the multiple-hops can be used instead of single-hop. The combination of cooperation and channel-adaptive routing is implemented for wireless communication in case of multiple-hop transmission.

With clustered wireless sensor network sensors within each cluster forward the message to another cluster via cooperative communication techniques. Only those sensors that correctly decode the packet from the source can take part in the following cooperative communication. Hence, the number of cooperating sensors is a random variable depending on both channel and noise realizations. In [47], authors develop an energy-efficient cooperative communication scheme for a clustered wireless sensor network. The scheme works in following ways primarily, the relay nodes are selected on a packet-by-packet basis and the number of relaying nodes is random. A sensor can act as one relay node only if it can decode the packet properly, which depends on both channel and noise realizations. Our packet-error-based analysis is more practical than existing symbol-error-based analysis, because packet errors can be detected via the cyclicredundancy-check (CRC) bits embedded in each packet [47]. Secondly, a multi-variable optimization problem to minimize the overall energy consumption, taking into accounts both the transmission energy and the circuit energy. Through numerical results, authors show that the total energy consumption can be considerably reduced by adjusting the transmit power for intra-cluster and intercluster transmission. In [47], authors also show that having more nodes in a cluster may be less energy efficient due to the extra circuit energy consumed by relay nodes. 


\section{CONCLUSION}

In this paper, we presented an overview on cooperative communication in wireless networks. We described the benefits of cooperative transmission than traditional non - cooperative communication. We talk about cooperative routing strategies in different networks. Here we present cooperative routing in wireless mesh networks, ad hoc networks and wireless sensor networks. We also discussed the advantages and applications of cooperative mesh network, Ad hoc networks and wireless sensor networks.

\section{REFERENCES}

[1] Theodore S. Rappaport, "Wireless Communications PRINCIPLES AND PRACTICE," Second Edition.

[2] A. Nosratinia, T.E. Hunter and A. Hedayat, "Cooperative communication in wireless networks," IEEE Communications Magazine, vol. 42, no. 10, October 2004, pp. 74-80.

[3] Jin Zhang and Qian Zhang, "Cooperative Routing in MultiSource Multi-Destination Multi-hop Wireless Networks," IEEE INFOCOM 2008 proceedings, pp. 306-310.

[4] Amir Khandani, Jinane Aboundi, Eytan Modiano, Lizhong Zheng, "Cooperative Routing in Static Wireless Networks," IEEE Transactions on Communications, to appear, 2008.

[5] Shoukang Zheng, "Performance Study of Cooperative Routing Metric for Multi-Hop Wireless Networks "IEEE, 2011.

[6] Stark C. Draper, Lingiia Liu, Andreas F. Molisch, and Jonathan S. Yedidia, "Cooperative Transmission for Wireless Networks Using Mutual-Information Accumulation," IEEE transactions on information theory, vol. 57, no. 8, August 2011, pp. $5151-5162$.

[7] Mohamed Elhawary and Zygmunt J. Haas, "EnergyEfficient Protocol for Cooperative Networks" IEEE/ACM Transactions on networking, vol. 19, no. 2, April 2011, pp. $561-574$.

[8] Ritesh Madan, Neelesh B. Mehta, Andreas F. Molisch and Jin Zhang, "Energy-Efficient Decentralized Cooperative Routing in Wireless Networks," IEEE TRANSACTIONS ON AUTOMATIC CONTROL, VOL. 54, NO. 3, MARCH 2009, PP. 512-527.

[9] Min Sheng, Jiandong Li, hongyan Li, and Yan Shi, "Small World Based Cooperative Routing Protocol for Large Scale Wireless Ad Hoc Networks," IEEE Communications Society, to appear, 2011.

[10] Zhiguo Ding and Kin K. Leung, "Cross-Layer Routing Using Cooperative Transmission in Vehicular Ad-hoc Networks" IEEE Journal on selected areas in communications, vol. 29, no. 3, March 2011, pp. 571-581.

[11] Youngchul Sung, Saswat Misra, Lang Tong, and Anthony Ephremides, "Cooperative Routing for Distributed Detection in Large Sensor Networks" IEEE journal on selected areas in communications, vol. 25, no. 2, February 2007, pp. $471-483$.

[12] Yun Li, Yanqiu Huang, Chonggang Wang, Xiaohu You, Ali Daneshmand, "A Cross-Layer Cooperative Method for IEEE 802.16 Mesh Networks," in IEEE/IFIP Network Operations and Management Symposium - NOMS 2010: Mini-Conference, PP. 17 - 23.
[13] Christian Ibars and Aitor del Coso. "Increasing the Throughput of Wireless Mesh Networks with Cooperative Techniques," IST project 27402, WIP.

[14] Behrouz A. Forouzan, "Data Communications and Networking", Fourth Edition.

[15] Gerhard Kramer, Ivana Mari'c and Roy D. Yates, "Cooperative Communications", Foundations and TrendsR_in Networking Vol. 1, Nos. 3-4 (2006) 271-425.

[16] T. M. Cover and A. A. E. Gamal, "Capacity Theorems for the Relay Channel," IEEE Trans. Info. Theory, vol. 25, no. 5, Sept. 1979, pp. 572-584.

[17] J.N. Laneman, "Cooperative Diversity in Wireless Networks: Algorithms and Architectures," Ph.D. Thesis, Massachusetts Institute of Technology, Aug. 2002, Cambridge, MA.

[18] J.N. Laneman, D.N.C. Tse, G.W. Wornell, "Cooperative diversity in wireless networks: Efficient protocols and outage behavior," IEEE Transactions on Information Theory, vol. 50, issue 12, Dec. 2004, pp. 3062-3080.

[19] J.N. Laneman, G.W. Wornell, "Distributed space-timecoded protocols for exploiting cooperative diversity in wireless networks," IEEE Transactions on Information Theory, vol. 49, issue 10, Oct. 2003, pp. 2415-25.

[20] Pei Liu, Zhifeng Tao, Zinan Lin, Elza Erkip, and Shivendra Panwar, "Cooperative Wireless Communications: a Cross-layer Approach," IEEE Wireless Communications, August 2006, pp. 84-92.

[21] Liping Wang and Viktoria Fodor "Cooperative Geographic Routing in Wireless Mesh Networks" Mobile Ad hoc and Sensor Systems (MASS), 2010 IEEE Page: 570 - 575.

[22] Yang Wendong, Cao Yueming, Xu Youyun "Wireless Cooperative Mesh Network: A New Architecture for Network Convergence" ZTE communication Magazine, 2008.

[23] Hicham Anouar, Christian Bonnet, Florian Kaltenberger, Raymond Knopp "OpenAirMesh-An Experimental Platform for Cooperative Mesh Networks" 1st COST2100 Workshop on MIMO and Cooperative Communications, June 3-4, 2008, Trondheim, Norway.

[24] Christian Ibars, Aitor del Coso "Increasing the Throughput of Wireless Mesh Networks with Cooperative Techniques" Mobile and Wireless Communications Summit, IEEE 2007. Page(s): $1-5$.

[25] Elzbieta Beres and Raviraj Adve" Cooperation and Routing in Multi-Hop Networks," IEEE-2007.

[26] Yun Li1,3, Yanqiu Huang1, Chonggang Wang2, Xiaohu You3, Ali Daneshmand" A Cross-Layer Cooperative Method for IEEE 802.16 Mesh Networks" Network Operations and Management Symposium (NOMS), IEEE, 2010 .

[27] PERKINS, C.E.: 'Ad-hoc networking' (Addison-Wesley, 2001).

[28] E. Royer, "A review of current routing protocols for ad hoc mobile wireless networks," IEEE Personal Communications, pp. 46-55, Apr. 1999.

[29] Xie Fang, Tian Hui, Yang Ning and Zhang Ping, "The Impact of Relaying Strategies on the Cellular System", In the 8th Wireless Word Radio Forum, Beijing, China, Mar.2004.

[30] J. Nicholas Laneman, David N. C. Tse, and Gregory W. Wornell, "Cooperative Diversity in Wireless Networks: Efficient Protocols and Outage Behavior", IEEE Trans. inform. theory.

[31] Xie Fang, Tian Hui, Zhang Ping,Yang Ning "Cooperative Routing Strategies in Ad hoc Networks" IEEE Vehicular Technology Conference, 2005. 
[32] Mihaela Cardei, Member, IEEE, Jie Wu, Senior Member, IEEE, and Shuhui Yang, "Topology Control in Ad Hoc Wireless Networks Using Cooperative Communication" IEEE Transactions on Mobile Computing, Volume: 5, Issue: $6,2006$.

[33] Min Sheng, Jiandong Li, hongyan Li, and Yan Shi "Small World Based Cooperative Routing Protocol for Large Scale Wireless Ad Hoc Networks" IEEE International Conference on Communications (ICC), 2011.

[34] Nam-Soo Kim, Beongku An, Do-Hyeon Kim, Ye Hoon Lee "Wireless Ad-hoc Networks Using Cooperative Diversity-based Routing in Fading Channel", IEEE Pacific Rim Conference on Communications, Computers and Signal Processing, 2007.

[35] Shoukang Zheng Shoukang Zheng "Performance Study of Cooperative Routing Metric for Multi-Hop Wireless Networks" IEEE Vehicular Technology Conference (VTC Spring), 2011.

[36] Fulu Li ,Kui Wu ,Andrew Lippman "Energy-Efficient Cooperative Routing in Multi-hop Wireless Ad Hoc Networks" 25th IEEE International Performance, Computing, and Communications Conference, 2006. IPCCC 2006.

[37] Liangzhong Yin and Guohong Cao "Supporting Cooperative Caching in Ad Hoc Networks", IEEE Transactions on Mobile Computing, Volume: 5, Issue: 1, 2006.

[38] Ching-Ling Huang, Yaser P. Fallah, Raja Sengupta Hariharan Krishnan "Information Dissemination Control for Cooperative Active Safety Applications in Vehicular Ad-Hoc Networks", IEEE Global Telecommunications Conference, 2009. GLOBECOM 2009.

[39] Zigui Yang, Jianhan Liu and Anders Høst-Madsen "Cooperative Routing and Power Allocation in Ad-hoc Networks" IEEE Global Telecommunications Conference, 2005. GLOBECOM '05.

[40] Youngchul Sung, Saswat Misra, Lang Tong, and Anthony Ephremides, "Cooperative Routing for Distributed Detection in Large Sensor Networks" IEEE Journal on selected areas in communications, vol. 25, no. 2, February 2007, pp. 471-483.

[41] Yao-Win Hong, Wan-Jen Huang, Fu-Hsuan Chiu, and C.C. Jay Kuo "Cooperative Communications in ResourceConstrained Wireless Networks", IEEE Signal Processing Magazine, Volume: 24, Issue: 3, 2007.

[42] Xiaoxia Huang, Member, Hongqiang Zhai, Member and Yuguang Fang, Fellow, IEEE "Robust Cooperative Routing Protocol in Mobile Wireless Sensor Networks", IEEE Transactions on Wireless Communications, Volume: 7, Issue: 12, Part: 2, 2008.

[43] Lichuan Liu, Zhigang Wang and MengChu Zhou, "Energy-Efficient Cooperative Routing for Wireless Sensor Networks Using Space Time Block Code", IEEE International Conference on Systems, Man and Cybernetics, 2007.

[44] Weiyan Ge and Junshan Zhang ,Guoliang Xue "Joint Clustering and Optimal Cooperative Routing in Wireless Sensor Networks" ICC 2008, IEEE International Conference on Communications, 2008.

[45] Liqian Luo, Chengdu Huang, Tarek Abdelzaher,John Stankovic "EnviroStore: A Cooperative Storage System for Disconnected Operation in Sensor Networks" 26th IEEE International Conference on Computer Communications, 2007.

[46] S.Sadasivam,M.Sheik Dawood,G.Athisha "A Modified Node Selection Scheme for Cooperative Wireless
Networks" International Journal of Computer Applications Volume 13- No.4, January 2011.

[47] Zhong Zhou1, Shengli Zhou, Shuguang Cui, and Jun-Hong Cui, "Energy-efficient cooperative communication in clustered wireless sensor networks", IEEE Military Communications Conference, 2006.

[48] Xiaofeng Tao, Xiaodong Xu, and Qimei Cui, "An overview of cooperative communications", IEEE Communications Magazine, Volume: 50, Issue: 6, Page(s): 65 - 71, June 2012.

[49] Qian Li, Rose Qingyang Hu, Yi Qian, Geng Wu, "Cooperative communications for wireless networks: techniques and applications in LTE-advanced systems ", IEEE Wireless Communications, Volume: 19, Issue: 2, Page(s): 22 - 29, April 2012.

[50] Quansheng Guan, F. Richard Yu, Shengming Jiang, Victor C. M. Leung, Hamid Mehrvar, "Topology Control in Mobile Ad hoc Networks With Cooperative Communications “, IEEE Wireless Communications, Page(s): 74 - 79, April 2012.

[51] Dan Wu, Yueming Cai, Liang Zhou, and Jinlong Wang, "A Cooperative Communication Scheme Based on Coalition Formation Game in Clustered Wireless Sensor Networks ", IEEE Transactions on wireless communications, vol. 11 , no. 3, page(s): $1190-1200$, March 2012.

[52] A. F. M. Shahen Shah and Md. Shariful Islam, "CSCM: A Contention Sensitive Routing Metric for Efficient Cooperative Communication in Multi-Hop Wireless Networks," (IJIDCS) International Journal on Internet and Distributed Computing Systems, Vol. 2 No. 2, 2012, pp. 157-163.

\section{Authors' Profiles}

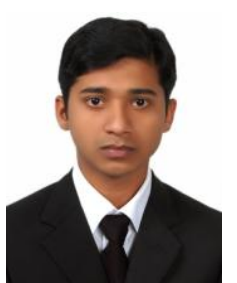

A. F. M. Shahen Shah received his Master in Information Technology (MIT) degree from IIT, University of Dhaka, Bangladesh in 2011. Now he is studying M.Sc. in Information and Communication Technology at IICT, Bangladesh University of Engineering and Technology (BUET), Bangladesh. His current research interest includes the design of routing protocols, metrics and wireless communication. He also worked on bandwidth optimization, frequency reuse schemes in mobile communication. He is a coauthor of two research papers and a book.

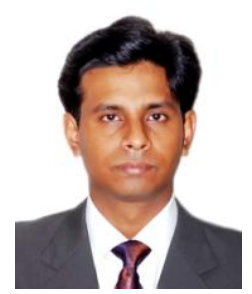

Md. Shariful Islam received his B.Sc. and M.Sc. degree in Computer Science from the University of Dhaka, Bangladesh, in the year 2000 and 2002, respectively. He completed his M.S degree in Information Technology from the Royal Institute of Technology (KTH), Sweden, in 2005. He obtained his Ph.D degree in Computer Engineering from Kyung Hee University, South Korea in February, 2011. He is now working as an Associate Professor in the Institute of Information Technology (IIT), University of Dhaka, Bangladesh. His current research interests include the design of routing protocols, metrics and MAC protocols for wireless mesh networks. He also worked on security issues related to Wireless AdHoc and Mesh Networks. He has published a good number of research papers in international conferences and journals. He is a member of IEEE and KICS. 